\title{
Creatividad, ¿recurso o riesgo?
}

María Aranguren

\section{Resumen}

En el presente trabajo se revisarán los aportes y perspectivas actuales más relevantes que abordan el tema de las relaciones entre creatividad, psicopatología y bienestar psicológico. Sabemos que la creatividad es un proceso, que desde lo psicológico, involucra e integra, distintos aspectos cognitivos, volitivos y emocionales de la persona. Además, la creatividad se asocia a una mayor fluidez de ideas, flexibilidad cognitiva, capacidad para resolver los problemas, originalidad (Guilford, 1956, 1968; Torrance, 1966); cualidades que llevarían a pensar en una mejor calidad de vida. Muchos psicólogos de la corriente humanista y otros desde la corriente psicoanalítica, rescatan a la creatividad como un elemento vinculado a la sublimación, al juego, al pensamiento simbólico, a la trascendencia y autorrealización (Maslow, Rogers, Winnicott, Frankl, Freud, entre otros). Sin embargo, muchos estudios también demuestran que los individuos altamente creativos tienen una mayor vulnerabilidad a padecer trastornos mentales, sobre todo en lo que respecta a los trastornos del estado de ánimo.

Los estudios actuales referentes a la creatividad y a la psicopatología, intentan esclarecer, tomando en consideración distintas perspectivas, cuáles son los posibles nexos entre estas dos entidades conceptuales. Entre las perspectivas y/o aportes, considerados para abordar la problemática se encuentran: (a) la perspectiva histórico-social, (b) los aportes de la psicometría y de psicología cognitiva, en relación a los rasgos de personalidad y estilos cognitivos, (c) la perspectiva "sistémica" o aportes desde la teoría de los fractales y (d) los aportes de la psicología positiva.

Palabras Clave: Creatividad - Psicopatología - Bienestar psicológico.

- Licenciada en Psicología. Docente. Facultad de Psicología y Educación. Pontificia Universidad Católica Argentina (UCA). E-mail: maria.aranguren@yahoo.com 


\section{Abstract}

The main objective of this work is to review some of the present perspectives and outstanding contributions that examine the relationship between creativity, psychopathology and psychological well-being. We know that creativity is a psychological process, which involves and integrates cognitive, volitional and emotional aspects of human behavior. In addition, creativity is associated with a greater flow of ideas, cognitive flexibility, ability to solve problems and originality (Guilford, 1956, 1968; Torrance, 1966), all qualities that lead to think of a better quality of life. Many of the humanist psychologists and others from the psychoanalytic stream stress that creativity is an element linked to sublimation, play, symbolic thought, transcendence and self-realization (Maslow, Rogers, Winnicott, Frankl, Freud, among others). However, many empirical studies show that highly creative individuals are more vulnerable to mental disorders, especially in the spectrum of mood disorders.

Some current studies attempt to clarify the issue of creativity and psychopathology, taking into account different perspectives, which may explain the possible links between these two conceptual entities. The perspectives or contributions, which are considered in the present article to address this problem, are: (a) the historical-social perspective, (b) the contributions of psychometric studies and cognitive psychology, in relation to personality traits and cognitive styles, (c) the "systemic" perspective or contributions from fractal geometry, and (d) the contributions from positive psychology.

Key Words: Creativity - Psychopathology - Psychological Wellbeing. 


\section{Introducción}

El término creatividad ha sido utilizado en diferentes ámbitos y de diferentes maneras a través de la historia. La creatividad es un proceso complejo en el cual intervienen factores psicológicos, biológicos y socio-culturales. Se dice que una persona es creativa cuando tiene ideas originales, novedosas, fuera de lo común; cuando tiene la capacidad de cuestionar la realidad previamente establecida y repensarla en términos diferentes o cuando una persona es imaginativa y fantasiosa. También, se dice que alguien es creativo cuando es capaz de resolver un problema de manera distinta a lo habitual. Otra de las acepciones, se utiliza para designar a aquellas personas que han realizado o realizan, contribuciones relevantes en el ámbito científico, artístico o empresarial. En esto último, se pone especial énfasis en los productos creativos que dichas personas generan.

En ocasiones, la creatividad suele ser confundida con la superdotación, el talento, el don o con la genialidad. En este sentido es de hacer notar que en la actualidad, la creatividad -a diferencia de la superdotación, el talento, el don o la genialidad- es considerada como un atributo que poseen todos los seres humanos, en mayor o menor medida. Mientras que, el talento o el don, hacen referencia a cierta capacidad innata para desarrollar tal o cual actividad, la creatividad, no lo implica necesariamente. Es decir, una persona con cierto talento para la música puede aprovechar esta condición, desarrollarla, formarse en este dominio del saber y, luego, ser un músico reconocido por su creatividad. Pero también, puede suceder que la persona no tenga esa destreza innata y simplemente elija desarrollarla, por un gusto particular, logrando lo mismos resultados que aquella persona especialmente dotada. La superdotación ha sido definida inicialmente por Terman, como un coeficiente intelectual superior a un nivel determinado (Carr, 2004/2007). Sin embargo, en la actualidad esta definición ha resultado un tanto insuficiente siendo reconsiderada y ampliada por autores como Renzulli y Gardner (Renzulli, 1986; Gardner, 1983/1993 citado en Carr, 2004/2007). A diferencia de la creatividad, los factores hereditarios o constitucionales tienen un papel predominante en el desarrollo de la superdotación. Aún así, muchos autores mencionan la importancia de los factores relacionados con el entorno y con la educación para su despliegue. A pesar de que la creencia popular tiende a afirmar que los niños prodigio crecerán como adultos creativos, esta sentencia no ha sido confirmada por la investigación. Según Carr (2004/2007), “la mayoría de los niños superdotados se convierten en adultos con éxito y bien adaptados que llegan a ser expertos en su campo, pero sólo una minoría de estos niños acaban siendo adultos creativos que hacen grandes innovaciones” (p. 194). Además, los estudios en referencia a los rasgos de personalidad muestran algunas diferencias notables entre los rasgos manifestados por niños superdotados y los niños creativos. En este 
sentido, Carr (2004/2007) señala que los niños superdotados "suelen mostrar conformidad y proceden de familias estables que ofrecen apoyo. En cambio, los adultos creativos suelen ser rebeldes y su infancia suele estar marcada por tensiones y dificultades que les predisponen a desafiar las reglas y las prácticas convencionales de su campo" (p. 194). Por último, la genialidad, que es un concepto bastante afín a la superdotación, implica un reconocimiento social aún mayor respecto del producto creativo realizado. Se podría decir que la genialidad implica un juicio social, cierta admiración y consagración del individuo en cuestión por el logro alcanzado. Indudablemente, todo genio ha de ser creativo, pero no todo creativo es considerado un genio.

En general, todas las personas estarían de acuerdo en decir que "ser creativo" es un atributo deseable e, incluso, hasta codiciado. La historia de la humanidad da cuenta de la importancia que tiene la creatividad para la supervivencia, el progreso de la cultura y el desarrollo de las sociedades. Todos los grandes cambios y aportes que se han dado en la historia, sea en el ámbito de las ciencias o de las artes, son producto del ingenio y la capacidad de personas creativas. Einstein, Newton, Galileo, Darwin, Leonardo da Vinci, Picasso, Beethoven, Dante Alighieri, Proust, Dostoievski, Freud, Nietzsche, Marx, por sólo nombrar algunos, fueron quienes realizaron aportes que conmovieron la manera, no sólo de pensar el mundo, si no de vivir en el mundo. Según Csikszentmihalyi (1996/1998), la creatividad "es un proceso por el cual dentro de la cultura resulta modificado un campo simbólico” (p. 23). Siguiendo esta línea de pensamiento, se puede observar que la creatividad es un área de suma relevancia en nuestras vidas, a pesar de que su estudio resulte a veces dificultoso y se necesite de un abordaje multifacético para poder entender este proceso tan complejo.

En la actualidad, se han realizado numerosos estudios en el área de la creatividad intentando esclarecer cuáles son los criterios de evaluación adecuados, cuáles son aquellos factores que promueven la creatividad, cuáles son aquellos factores que la dañan, qué rasgos de personalidad caracterizan a los individuos creativos, entre otras cuestiones.

El objetivo de este artículo es realizar una revisión bibliográfica sobre las publicaciones más relevantes que abordan el tema de las relaciones entre creatividad, psicopatología y bienestar psicológico. En otras palabras, sabemos que la creatividad es un proceso, que desde lo psicológico, involucra e integra, distintos aspectos cognitivos, volitivos y emocionales de la persona. Además, la creatividad se asocia a una mayor fluidez de ideas, flexibilidad cognitiva, capacidad para resolver los problemas, originalidad (Guilford, 1956, 1968; Torrance, 1966), todas cualidades que llevarían a pensar en una mejor calidad de vida. Muchos psicólogos de la corriente humanista y otros de la psicoanalítica, rescatan la creatividad como un elemento vinculado a la sublimación, al juego, al pensamiento simbólico, a la 
trascendencia y a la autorrealización (Maslow, Rogers, Winnicott, Frankl, Freud, entre otros). Sin embargo, muchos estudios también demuestran que los individuos altamente creativos tienen una mayor vulnerabilidad a padecer trastornos mentales, sobretodo en el espectro de los trastornos del estado de ánimo. Otros estudios arrojan evidencia sobre cierta relación entre la dimensión de psicoticismo, como rasgo de personalidad y creatividad. Entonces, la pregunta que nos hacemos es: ¿la creatividad es un recurso o un riesgo?

\section{Perspectivas actuales sobre la creatividad}

Desde la Antigüedad, la idea de genio ha sido asociada a la idea de locura. Los estudios actuales en referencia a la creatividad y la psicopatología, intentan esclarecer, tomando en consideración distintas perspectivas, cuáles son los posibles nexos entre estas dos entidades conceptuales. Entre las perspectivas y/o aportes considerados para abordar la problemática se encuentran: (a) la perspectiva histórico-social, (b) los aportes de la psicometría y de psicología cognitiva, en relación a los rasgos de personalidad y estilos cognitivos, (c) la perspectiva "sistémica" o aportes desde la teoría de los fractales y (d) los aportes de la psicología positiva.

\section{II.1. Perspectiva histórico-social.}

En un estudio realizado por Becker (2000-2001) se plantea la decisiva importancia que tuvo el Romanticismo sobre las concepciones actuales que tenemos con respecto a la relación entre genialidad y locura. Becker (2000-2001) señala que no fue sino hasta el movimiento romántico en la literatura en que la locura se comenzó a ver como una condición sine qua non para la genialidad en los ámbitos artísticos.

Tanto la idea de genio y la idea de locura fueron variando en las distintas épocas de la historia -en la Antigüedad, en el Renacimiento Italiano, en el Siglo de las Luces - según las características socio-culturales de cada una.

Para poder entender la concepción de los antiguos griegos sobre la naturaleza del fenómeno creativo, hay que tener en consideración dos conceptos básicos que son: el de "posesión demoníaca” y el de melancolía. Según la visión socrática, la posesión demoníaca era considerada como un regalo divino concedido sólo a unos pocos individuos. El poeta, el sacerdote, el filósofo y el sabio sólo eran capaces de comunicarse con los dioses mediante la intervención del demonio. Esta concepción de los demonios como representantes de la beneficencia de los dioses, fue aceptada por Platón y otros filósofos, así como también, encuentra apoyo en la doctrina de la Divina Locura o enthousiasmos. Desde este punto de vista, el poeta, 
desprovisto de talento propio, sería inspirado por los dioses y, representante de los propósitos divinos.

Por otro lado, la afirmación aristotélica de que el talento extraordinario está caracterizado por un temperamento melancólico, ha sido malinterpretada por muchos contemporáneos. Para Aristóteles, la enfermedad mental no se daba en todos los casos de melancolía. Más bien, según su reformulación de la teoría humoral de Hipócrates, el término melancolía era utilizado para describir un tipo de individuo, el homo melancholicus, que, dependiendo de la particular mezcla de humores, podía ser considerada más sana o enferma (Wittkower \& Wittkower, 1963 en Becker, 2000-2001).

Es a partir del Renacimiento Italiano que comienza a utilizarse el término de "genio" o "genialidad" para denominar a las personas especialmente dotadas en determinados aspectos artísticos o creativos. Sin embargo, la creatividad era concebida, principalmente, en términos de imitación de los "grandes maestros" o de las obras de la naturaleza. El producto creativo respondía entonces, no a la imposición del autor sobre su obra, sino a la influencia de los grandes predecesores o de la naturaleza misma, sobre la obra. A diferencia de la concepción moderna del genio, la cual subraya por sobretodo la originalidad como la característica más deseada del individuo creativo, para la tradición humanista lo importante era la imitación ideal, la copia perfecta o el reflejo idóneo de lo que ya estaba dado en la naturaleza.

Hasta finales del siglo XVIII, prevalece la concepción de la Ilustración en donde se consideraba el acto creativo como resultado de un equilibrio entre todas las facultades humanas. El genio era un individuo bien educado, con mucha imaginación templada por un buen gusto, que había aprendido de los grandes clásicos de la historia y debía su reconocimiento a los maestros.

A finales del siglo XVIII y principios del siglo XIX, bajo el impacto del Romanticismo, se dio un profundo cambio respecto de la consideración prevalente del concepto de "genio". Este cambio estaba íntimamente relacionado con la desaparición de la clase social tradicional -la nobleza y la burguesía aristocráticaque sostenía la producción artística. El artista quedo despojado de sus seguridades habituales, viéndose obligado a introducirse en los inestables mercados modernos. Generalmente pobres y privados de poder político durante la mayor parte del siglo XIX, los artistas u hombres de letras se sentían sumidos en una masa anónima. A pesar de que la idea de genio todavía otorgaba cierto respeto y, hasta incluso, reverencia, los hombres de genio carecían de una identidad propia que los separase eficazmente del anonimato de las masas, y que les permitiera hacer frente al mundo de una manera eficaz.

De esta manera, los artistas románticos se vieron obligados a tratar de establecer una identidad firme, que los consolidara como un grupo social 
independiente de sus antepasados y los singularizara frente a sus contemporáneos. En este sentido, el genio no podía seguir siendo considerado en los términos planteados por el Iluminismo. De ahí que, los románticos, concedieran un rol predominante a la imaginación, dejando de lado todo tipo de equilibrio entre las facultades mentales. Enfatizando el papel de la imaginación en el acto creativo, los artistas lograron, simultáneamente, separarse de las premisas establecidas en el pasado, lo mismo que adquirir cierto margen de libertad intelectual o independencia para actuar según sus propias ideas. Sin embargo, esta nueva adquisición y los cambios logrados, no fueron gratuitos para los artistas. A pesar de que la nueva definición liberaba a los artistas del pasado, también los separaba de los atributos que antes habían servido para protegerlos y salvaguardarlos. Es decir, mediante la concepción anterior del genio, los hombres ilustrados eran considerados como equilibrados, sabios, personas en las que la salud era resultado de un minucioso equilibrio de sus facultades mentales. Al hacer prevalecer la imaginación sobre cualquier otra facultad mental, los románticos destituyeron ese equilibrio estableciendo, al mismo tiempo, un lógico fundamento para la asociación entre genio y locura. De manera irónica, los mismos artistas quedaron atrapados en su propia lógica al comenzar a sospechar que no había otra posibilidad que, de hecho, aceptar inevitablemente la locura, para llegar a ser un genio.

A pesar de todo lo dicho hasta aquí, sería erróneo asumir que todos esos temores y auto-reconocimientos de los artistas, eran suficientes para establecer un veredicto médico sobre la relación entre locura y genio. En realidad, hasta mediados del siglo XIX, la cuestión permanece más como una especulación de tipo filosófica que como una aseveración de tipo científico.

Sintetizando, se podría decir que la asociación entre creatividad y locura tuvo sus orígenes en la Antigüedad y recibió una nueva interpretación, así como también un reforzamiento, en la época del Romanticismo. De la misma manera en que, actualmente, se espera que los científicos y los ingenieros demuestren las cualidades de objetividad, razonabilidad y estabilidad emocional, se espera encontrar en los artistas una gran intuición, imaginación, ciertos rasgos extravagantes, facilidad de expresión emocional, sensibilidad, irritabilidad o excitabilidad, en suma, una especie de locura acotada. No sería para nada erróneo asumir entonces, que mientras estas expectativas sigan siendo parte de la ideología profesional dominante, tanto los escritores como los artistas contemporáneos, fomentarán dichas características en vez de desalentarlas.

Todo lo dicho hasta aquí, no pretende afirmar que los síntomas psicopatológicos que manifiestan algunas personas creativas sean, nada más, que el resultado de la adherencia a un rol social establecido. Más bien, lo que se busca mediante esta reflexión, es que en las investigaciones actuales sobre el tema, se tengan en cuenta las profundas implicancias que tienen los acontecimientos históricos en los 
desarrollos socioculturales, así como también, su impacto sobre las concepciones actuales acerca de la naturaleza del genio y el proceso creativo. Cualquiera sea la relación existente entre lo hereditario, la salud mental y la creatividad, las definiciones sociales -rol o identidad social- deben ser tenidas en cuenta como parte del debate si es que se quiere llegar a tener una visión global de la cuestión.

\section{II.2. Aportes de la psicometría y de la psicología cognitiva}

\section{II.2.1. Rasgos de personalidad}

Los estudios en referencia a los rasgos de personalidad relacionados con la creatividad tienden a mostrar resultados diversos y, en ocasiones, contradictorios. Sin embargo, algunas investigaciones arrojan evidencia sobre una posible relación entre los rasgos de psicoticismo y creatividad. Estos aportes vienen dados por estudios psicométricos en los que se incluyen distintos instrumentos de evaluación de la personalidad, como pueden ser: el Inventario de Multifacético de Personalidad de Minnesota (MMPI) y el Cuestionario de Personalidad de Eysenck (EPQ). Lo que se evidencia en estos estudios es que, en general, los individuos altamente creativos puntúan un nivel mayor al normal en las dimensiones asociadas a psicopatología (Barron, 1963 en Simonton, s.f.). Por ejemplo, la creatividad se encuentra positivamente correlacionada con las puntuaciones de psicoticismo en el EPQ (Eysenck, 1993, 1994, 1995).

En consonancia con lo anterior, y a partir de los estudios psicométricos realizados en el área, se destacan dos conjuntos de explicaciones teóricas para entender la naturaleza de las relaciones entre la creatividad y los rasgos de psicoticismo (Simonton, S.f.).

Por un lado, a pesar de que los individuos altamente creativos tienden a exhibir puntuaciones elevadas en determinadas escalas de medición clínica de psicopatología, las puntuaciones encontradas raramente son tan altas como para representar la presencia de un cuadro psicopatológico en sí. En lugar de ello, las puntuaciones oscilan entre los rangos normales y anormales (Barron 1963, Eysenck 1995 en Simonton S.f.). Por otro lado, es de hacer notar que, las puntuaciones superiores al promedio, en la dimensión de psicoticismo, están asociadas a rasgos de personalidad como la independencia y la disconformidad (Eysenck, 1995). Más aún, las puntuaciones elevadas en la dimensión de psicoticismo se relacionan con la capacidad de atención no selectiva, permitiendo así que aquellas ideas que normalmente serían filtradas durante el procesamiento de la información, entren en la mente. Este modo menos restrictivo de procesar la información está también asociado con la apertura a la experiencia, rasgo característico de las personas creativas (Peterson \& Carson, 2000; Peterson, Smith \& Caron, 2002). 
En segundo lugar, los individuos creativos presentan puntuaciones altas en otras características que parecerían amortiguar los efectos de algunos síntomas psicopatológicos. Particularmente, los sujetos creativos suelen presentar altos niveles de autosuficiencia y fortaleza yoica (ego-strength) (Barron, 1963; Cattell \& Butcher, 1968). De manera paralela a lo anterior, los individuos creativos parecerían ejercer cierto control meta-cognitivo sobre sus propios síntomas, aprovechando sus pensamientos bizarros en vez de quedar sujetos a ellos. Esto se encuentra favorecido por la presencia de una inteligencia superior a la media que les permite superar algunos inconvenientes que en otras circunstancias no sería posible.

\section{II.2.2. Estilos cognitivos}

Diferentes estudios han constatado algunas similitudes en la manera de procesar la información entre los individuos creativos y los individuos psicóticos.

Uno de los rasgos que caracteriza a las personas altamente creativas, es la manera particular en que perciben y experimentan el mundo. De manera paralela, uno de los rasgos que caracteriza a los individuos con diagnóstico de psicosis es la manera en cómo procesan e interpretan la información del entorno (distorsión del pensamiento). De aquí, se puede decir entonces, que un rasgo que comparten tanto los individuos creativos como los psicóticos, es su manera particular o desviada, según el caso, de decodificar y procesar la información del mundo exterior e interior (Prentky, 2000-2001).

Las similitudes entre los estilos de pensamiento de sujetos normales y psicóticos han sido trabajados en detalle por McConaghy (1960, 1961; McConaghy \& Clancy, 1968). McConaghy (1960) distinguió entre dos modelos de procesamiento de la información que podían reflejar una predisposición a la psicosis. En el primer caso, los mecanismos de filtro se encuentran dañados, de ahí que, se produzca una intrusión de asociaciones irrelevantes en el procesamiento de la información. Los procesos de pensamiento se tornan vagos y pasan a ser dominados por la intuición. Este trastorno parece afectar al $10 \%$ de la población y puede implicar cierta vulnerabilidad a padecer de esquizofrenia. En el segundo caso, lo que está dañado es la capacidad para realizar atribuciones del tipo lógico-causal. Es decir que, una vez que se ha alcanzado una conclusión, la persona adhiere a esa idea de manera absoluta. En este sentido, se dice que en el individuo psicótico la lógica triunfa sobre el sentido común, condición que puede predisponer a la paranoia.

McConaghy (1961) denominó, al tipo de pensamiento predominante en los sujetos esquizofrénicos, como pensamiento alusivo y propuso que este tipo de pensamiento podía ser observado en una variedad de personas, de las cuales algunas nunca habían padecido de un trastorno esquizofrénico. El pensamiento alusivo estaría caracterizado por su imprecisión (demasiado abstracto) y un 
discurso inapropiado, en el que predomina una inclusión exagerada de elementos que habitualmente serían filtrados por la atención, permitiendo de esta manera asociaciones remotas y hasta, a veces, absurdas (overinclusion). En cierta forma, podríamos decir que este pensamiento es similar a la disfunción que Marietan (1996) denomina disgregación del pensamiento, el cual está caracterizado por una alteración del curso de pensamiento causado por un debilitamiento progresivo de la idea directriz, en donde la estructura del pensamiento se pierde o se relaja.

En consonancia con lo anterior, es de resaltar, que hay una gran semejanza entre los estilos cognitivos que caracterizan a los sujetos creativos definidos por Cattell y sus colegas y aquellos estilos que caracterizan a los psicóticos definidos por McConaghy. En el primer caso, el resultado es un producto creativo o pensamiento creativo mientras que en el segundo, se da un trastorno del pensamiento que podría ser llamado hiperinclusión o superinclusión (overinclusion). La supuesta diferencia entre el pensamiento divergente, la alta capacidad de asociación de ideas, la irrelevancia de las conexiones temáticas en los psicóticos y los saltos conceptuales, la flexibilidad cognitiva y los afortunados descubrimientos en los artistas y científicos, debería ser revisada en mayor detalle. En este sentido, varios estudios realizados han postulado diversas explicaciones para dar cuenta de las semejanzas encontradas entre los estilos cognitivos de los sujetos psicóticos y los estilos cognitivos de los individuos creativos. Una de las propuestas más interesantes es la de Claridge (1972) y Prentky (2000-2001), quienes sugieren la existencia de un continuo entre el curso de pensamiento normal y el curso de pensamiento patológico. Según Claridge (1972), un trastorno de pensamiento no debería ser evaluado sólo por su presencia o ausencia, sino que se debería tener en cuenta una gama de grises entre un curso de pensamiento normal y uno patológico. Más aún teniendo en consideración que algunos rasgos de pensamiento psicótico se encuentran presentes también en sujetos de población general, sólo que de manera atenuada y sin causar otros tipos de déficit a nivel mental (Prentky, 2000-2001).

El modelo teórico propuesto por Prentky (2000-2001) sugiere que el procesamiento de la información se constituye como un continuo en el que hay dos extremos: aquellas personas que tienden hacia la expansión y aquellas que tienden hacia la constricción. La superposición entre los sujetos altamente creativos y aquellos que padecen de enfermedades mentales puede encontrarse en los desvíos de este continuo. Cualquier desviación exagerada hacia alguno de los extremos, correspondería a los sujetos que padecen de algún trastorno del pensamiento, mientras que la creatividad vendría a estar favorecida por una desviación óptima sin llegar a posicionarse en ninguno de los extremos. Cuando esta desviación "óptima" se desliza hacia alguno de los extremos del continuo, lo que hasta ese entonces era normal, se torna patológico. La noción de un "desvío óptimo” se 
encuentra implícita en Ornstein (1997) quien señala que entre los parientes de individuos esquizofrénicos se pueden encontrar personas altamente creativas. Según su parecer, esto podría indicar que el pensamiento de tipo desorganizado, en pequeñas cantidades, puede ser muy útil, aunque, en grandes cantidades, puede resultar nocivo para la salud mental.

\section{II.3. Perspectiva sistémica. Teoría de los fractales}

En esta perspectiva que he dado el nombre de sistémica, se hará referencia a las diferencias encontradas entre los artistas y los científicos creativos en relación a la psicopatología presentada por estos grupos. Un número considerable de estudios recientes han documentado una alta frecuencia de enfermedades mentales en artistas creativos (Ludwig, 1992, 1994, 1995; Andreasen, 1987; Jamison, 1993; Post, 1994, 1996; Richards, 1981, citados en Ludwig, 1998). Sin embargo, estos resultados no son replicados cuando la población de estudio implica a científicos reconocidos a nivel internacional por sus aportes. Es decir, no hay diferencias en los logros creativos realizados por estos grupos, sea que tomemos en consideración artistas o científicos, sin embargo, sí se encuentran diferencias notables en cuanto a la vulnerabilidad que presentan cada uno de los grupos mencionados, a padecer una enfermedad mental. En un estudio realizado por Ludwig (1998) se intenta dar respuesta a las desigualdades encontradas entre el ámbito artístico y el ámbito científico, aplicando algunas nociones de la teoría de los fractales de Mandelbrot (1988).

Algunos años atrás, Benoit B. Mandelbrot (1988), inventó una nueva forma de matemática, conocida como geometría fractal, que es aplicada a ciertos objetos naturales como costas marítimas, copos de nieve, rocas u olas. El término fractal describe cualquier tipo de estructura geométrica que comúnmente exhibe la propiedad de autosimilitud. "Según Mandelbrot, un objeto es autosimilar o autosemejante si sus partes tienen la misma forma o estructura que el todo, aunque pueden presentarse a diferente escala y pueden estar ligeramente deformadas" (Wikipedia). Esta reiteración de detalles o patrones irregulares ocurre de manera progresiva en escalas menores y puede, en el caso de entidades puramente abstractas, continuarse indefinidamente, de manera que cada parte de cada parte, siendo magnificada, podrá verse básicamente como el mismo objeto total. En efecto, un objeto autosimilar se mantendrá invariante bajo cambios de la escala esto es, escalamiento simétrico-.

Lo que plantea Ludwig (1998) en su estudio es que si las nociones de Mandelbrot acerca de los objetos de forma irregular, son válidas para cualquier forma irregular, de manera metafórica, entonces, esta noción también debería ser reflejada en las relaciones entre enfermedad mental y creatividad. Así sería 
esperable encontrar diferencias comparables entre las ciencias y el arte como un todo, a nivel macroscópico, como también a nivel microscópico, lo que es, entre las distintas subcategorías de profesiones dentro de cada ámbito sea este científico o artístico. El mismo patrón debería repetirse ya sea en el caso de las ciencias como en el casos de las artes. Las personas que trabajan en campos que requieren de mayor precisión, replicación, formalidad y objetividad deberían mostrarse más estables emocionalmente que aquellas otras personas que se dedican a áreas diferentes; de manera contraria a lo anterior, las personas que trabajan en ámbitos donde se enfatiza la subjetividad, el significado y el valor personal, deberían mostrarse más inestables emocionalmente.

Efectivamente, los resultados hallados por Ludwig se encuentran en consonancia con lo planteado. En un primer nivel de observación se constata que los profesionales del área artística suelen presentar mayores índices de enfermedad mental que los profesionales del ámbito científico. En un segundo nivel -magnificación x 2, como él le llama- si nos focalizamos sólo teniendo en consideración el grupo de científicos y comparamos los científicos naturales con aquellos investigadores de las ciencias sociales, se constata el mismo patrón. Es decir, aquellos científicos que se desenvuelven en el ámbito de las ciencias "blandas" presentan una tasa de prevalencia de enfermedad mental dos veces mayor a la encontrada en los científicos de las ciencias duras (1992, 1995). Entonces, sería posible decir que aquellas profesiones que requieren de mayor objetividad, racionalidad, precisión van a presentar una menor asociación al riesgo de padecer una enfermedad mental, mientras que a medida que nos acercamos a las profesiones donde se enfatizan la subjetividad, la propia experiencia y el significado personal, se manifestará una mayor vulnerabilidad a presentar algún tipo de psicopatología asociada (Ludwig, 1998).

Ahora si se toma en consideración las artes, ya habiendo dejado en claro que en las artes hay un porcentaje mucho mayor de personas que padecen de trastornos emocionales, cabe esperar, en concordancia con lo planteado, que se encontrarán en determinadas orientaciones artísticas -aquellas que requieren de mayor objetividad, racionalidad y modos más precisos de expresión- tasas menores de enfermedad mental que en aquellas otras orientaciones artísticas donde los requisititos predominantes estén dados por lo emotivo, lo intuitivo o los modos más imprecisos de expresión. En un estudio previo realizado por Ludwig (1995), fueron comparadas las tasas de prevalencia de diferentes trastornos mentales en seis profesiones artísticas: arquitectura/diseño, artes visuales, composición musical, interpretación/ejecución musical, teatro y escritura. Se encontró que la prevalencia de cualquier forma de trastorno mental, no sólo varía a lo largo de las diferentes profesiones artísticas, sino que hay un incremento progresivo desde las profesiones caracterizadas por una mayor estructuración y precisión -como arquitectura y 
diseño -hasta llegar a aquellas caracterizadas por la subjetividad y la imprecisión, como las artes visuales y la escritura.

En un nivel de magnificación x 3, focalizándose en determinados estilos dentro de cada profesión, Ludwig (1998) propone dividir la profesión literaria en tres orientaciones de acuerdo a su nivel de estructuración: (a) ensayo o crítica literaria, periodismo; (b) ciencia ficción y (c) poesía. De manera consistente con la teoría fractal, cabría esperar que aquellas formas literarias como la poesía -donde predominan los elementos subjetivos, personales y auto-reveladores- mostraran una mayor incidencia de trastornos mentales que aquellas formas literarias como las biografías, la critica literaria o el periodismo, donde predominan elementos más analíticos, lógicos y prácticos. Los escritores de ficción, que combinan ambos tipos de elementos, deberían mostrar tasas de incidencia intermedias. A pesar de que los resultados hallados por Ludwig (1998) se orientan en esta dirección, no alcanzan un nivel estadísticamente significativo como para ser generalizadas las conclusiones. Sin embargo, sí se pudieron observar diferencias altamente significativas en referencia a la vulnerabilidad a padecer depresión entre los diferentes grupos.

Una de las conclusiones del estudio realizado por Ludwig (1998) es que no existe una relación entre creatividad y enfermedad mental per se, sino entre la presencia o ausencia de enfermedad mental en formas particulares de expresión creativa. Utilizando la metáfora del fractal, se observó que a nivel más microscópico se encontraban los mismos patrones que los hallados a nivel macroscópico.

El patrón dominante parece sugerir que mientras más elementos formales, naturales, matemáticos y objetivos predominen, menor es la prevalencia de enfermedad mental entre los miembros pertenecientes a dichas profesiones; mientras que a mayor cantidad de elementos que impliquen lo emotivo, lo personal, lo subjetivo, mayor prevalencia de enfermedad mental.

Lo que las observaciones realizadas por Ludwig (1998) también sugieren es que parecería existir una compleja relación entre los individuos creativos y sus profesiones. Aparentemente los individuos creativos tendrían en cuenta, al momento de elegir el ámbito de desempeño profesional, la profesión y la subprofesión, aquellos requisitos necesarios dentro de cada expresión creativa que mejor se adapte su propio temperamento y particular estilo cognitivo.

\section{II.4. Aportes de la Psicología Positiva}

En diferentes estudios se ha mostrado que determinados rasgos de personalidad son adecuados para predecir criterios globales de salud (Judge, Erez, Bono y Thoresen, 2002; Prenda y Lachman, 2001; Wasylkiw y Fekken, 2002). En este sentido, el concepto de creatividad es tratado, hoy en día, a nivel internacional y 
nacional, como un sinónimo de salud mental (Camacho, J. M., 2005; Contini de González, 2000; Martinez, S.f.; Rey \& Martinez, 1989; Wechsler y Nakano, 2002). Diversas investigaciones en el área de la creatividad indican la importancia que tiene la misma en el desenvolvimiento del pensamiento y actitudes más creativas, así como también, en el aumento de la motivación sobre diferentes aspectos del rendimiento académico y laboral, demostrando, de esta manera, un considerable impacto sobre aspectos como la satisfacción personal y la calidad de vida de los sujetos involucrados (Wechsler, 2001).

Por otro lado, Sternberg y Lubart (1995/1997) desde la teoría de la inversión, han señalado que las personas creativas presentan, en general, un conjunto de rasgos de personalidad más o menos estables a lo largo de la vida, tales como: perseverancia frente a los obstáculos, voluntad para crecer, tolerancia a la ambigüedad, apertura a la experiencia, voluntad para asumir riesgos, un fuerte autoconcepto así como también un fuerte sentimiento de convicción en sus propias creencias. Estando, muchos de estos rasgos mencionados, asociados a un buen funcionamiento psicológico y social.

Desde la Psicología Positiva, se han realizado numerosas investigaciones sobre la creatividad y la incidencia que tiene este proceso sobre las vidas de las personas. Según Csikszentmihalyi (1990/1997, 1996/1998) una de las experiencias que forma parte de cualquier acto creativo, es la experiencia del fluir (flow). El concepto del fluir fue formulado por Csikszentimihalyi (1990/1997, 1996/1998) quien lo definió como un estado en el que la persona se encuentra completamente absorta en la actividad que esta realizando, sin noción del paso del tiempo, simplemente disfrutando de hacer lo que en ese momento está haciendo. En su libro: Fluir. Una psicología de la felicidad (1990/1997) define el fluir o flujo, como una experiencia óptima, o un estado óptimo de experiencia interna que se caracteriza por dar orden a la conciencia. Este estado se logra mediante la combinación de distintos elementos -cognitivos, volitivos y emocionales- que permiten llegar a un estado de control sobre la conciencia. Según Csikszentimihalyi, (1990/1997) "contrariamente a lo que tendemos a suponer, el estado normal de la mente es el caos" (p. 182) y "la entropía es el estado normal de la conciencia” (p. 183). Es decir que las personas estamos constantemente tratando de poner orden a nuestros pensamientos, ya sea mediante la imposición del orden externo o mediante el entrenamiento de hábitos internos. Un ejemplo del primer tipo, podría ser cuando las personas se sientan a ver televisión, tratando de focalizarse en una actividad que no les demande mucha energía pero tampoco les permite sentir disfrute. En el caso del segundo tipo, encontramos muchas actividades que se encuentran en relación a la capacidad de sentir experiencias óptimas, como pueden ser la lectura, la escritura, el yoga, el deporte, escuchar o componer música, tocar un instrumento, pintar, socializar o charlar con otras personas, entre muchas otras. 
Muchas actividades son capaces de proveer disfrute. Sin embargo, Csikszentimihalyi (1990/1997), señala la necesidad de diferenciar el placer del disfrute, "el placer es un sentimiento de satisfacción que uno logra cuando la información en la conciencia nos dice que hemos conseguido cumplir con las expectativas controladas por los programas biológicos o por el condicionamiento social” (p. 77). Además, advierte que el placer es un componente importante de la satisfacción vital, pero por sí mismo no trae felicidad, ni suma complejidad o riqueza a nuestra personalidad ni tampoco se equipara a un crecimiento psicológico. Mientras que, el disfrute, sí se relaciona con la posibilidad de novedad, de cambio y de realización personal.

En la fenomenología del disfrute se destacan ocho elementos principales, a saber: (a) implica una actividad desafiante que requiere de habilidades específicas, (b) combina la acción y la conciencia, (c) hay metas claras en la actividad que se está realizando, (d) existe una retroalimentación entre lo que uno realiza y lo que uno obtiene, (e) toda nuestra concentración y atención se encuentra centrada en la tarea actual, (f) existe un sentimiento paradojal respecto del control que uno tiene sobre la actividad que se está realizando, (g) hay una pérdida de la autoconciencia y (h) hay una transformación en la percepción del paso del tiempo (Csikszentimihalyi, 1990/1997).

En cierta forma, esta posibilidad de ejercer el control sobre las propias experiencias y sobre nuestra conciencia, esta sensación de poder suspender la entropía mental, explica el por qué las actividades de flujo pueden resultar adictivas y estar asociadas, en ciertas ocasiones, a la presencia de psicopatología. Csikszentimihalyi (1990/1997), pone algunos ejemplos, como Bobby Fischer y Paul Morphy, quienes "llegaron a sentirse tan cómodos en el mundo lógicamente ordenado del ajedrez, que volvieron la espalda al desorden del mundo real” (p. 101). Entonces, las actividades de flujo tienen un potencial negativo, debido a la experiencia agradable resultante de su ejercicio. Por un lado, son capaces de mejorar la calidad de la experiencia por el orden mental que generan; por otro, pueden llegar a producir una adicción si la personalidad se convierte en prisionera de este orden y no puede, o no desea, enfrentarse a las ambigüedades implicadas en la vida cotidiana. Esto llevaría a un desequilibrio de las facultades o habilidades del individuo, como así también, a un desequilibrio en las actividades en las cuales se involucra el sujeto, cercenando, de esta manera, otros aspectos de la personalidad que deberían ser desarrollos para llegar a la armonía.

Csikszentimihalyi (1990/1997) señala también la asociación que se ha encontrado entre los escritores, poetas, y la psicopatología, ya que suelen presentar de manera frecuente, especialmente, síntomas de depresión y de desórdenes afectivos. Su propuesta explicativa para este fenómeno es que quizá, los escritores, justamente, han decidido volcarse a la escritura debido a un nivel excesivo de 
entropía mental, por lo que la escritura se vuelve un medio para otorgar orden a la conciencia, un método esclarecedor de las ideas, una terapia creada con el fin de proporcionar claridad a los sentimientos. En palabras de Csikszentimihalyi (1990/ 1997):

Es posible que los escritores únicamente puedan experimentar flujo creando mundo de palabras donde puedan actuar con soltura, borrando de la mente la existencia de una realidad problemática. Como cualquier otra actividad de flujo, escribir puede convertirse en una adicción y llegar a ser peligroso: fuerza al escritor a sujetarse a una gama limitada de experiencias y excluye otras opciones de enfrentarse a los acontecimientos. Pero cuando la escritura se usa para controlar la experiencia, sin dejar que controle la mente, es una herramienta de infinita sutileza y ricas recompensas (p. 201).

Ahora bien, otra salvedad que es necesario realizar, es que no todas aquellas actividades que nos proporcionan este sentimiento de flujo, son saludables. Las drogas, la violencia, el juego compulsivo, la promiscuidad sexual, pueden resultar para algunas personas experiencias agradables, sin embargo, esos episodios de fluir no constituyen una sensación de satisfacción y felicidad duradera. Como ya se ha señalado, no todas las actividades que dan placer son creativas ni representan una experiencia óptima, muchas de ellas pueden llevar a la adicción y a la entropía.

En su libro Creatividad: el fluir y la psicología del descubrimiento y la invención, Csikszentmihalyi (1996/1998) entrevista a más de noventa personas que tienen en común haber sido reconocidas por su creatividad en diferentes dominios del conocimiento. Los criterios de selección de muestra a tener en cuenta en el estudio mencionado, fueron: (a) la persona tenía que haber modificado un campo importante de la cultura (ciencias, artes, negocios, administración del Estado o bienestar humano en general), (b) tenía que estar aún activamente entregada a ese campo, (c) debía tener al menos sesenta años. Csikszentmihalyi señala que una característica que se repetía en todas las personas entrevistadas era que disfrutaban realmente haciendo su trabajo. Más allá del éxito alcanzado o de la remuneración económica, estas personas se mostraban sumamente reconfortadas por su propio trabajo y el desafío que éste implicaba. Csikszentmihalyi rescata que todos los entrevistados aludían a esta experiencia placentera en la realización de su trabajo, a la que él ha dado el nombre de flujo o fluir.

En referencia a la relación entre el fluir y la felicidad, Csikszentmihalyi puntualiza que aunque similares entre sí, la experiencia del fluir no es lo mismo que el sentimiento de satisfacción o felicidad. Es decir, cuando un escritor o un científico se sienten totalmente compelidos e involucrados en su trabajo, no se siente feliz, al menos, no se siente feliz sin perder el hilo de su pensamiento. El fluir 
se encuentra relacionado con la actividad misma, mientras que la felicidad o el placer, es un estado que sobreviene al finalizar o al interrumpir dicha actividad. En palabras de Csikszentmihalyi (1996/1998):

Sólo después de que el fluir se nos acaba, al final de una sesión o en momentos de distracción dentro de ella, podemos permitirnos el lujo de ser felices (...). A la larga, cuanto más fluido experimentamos en la vida diaria, más probable es que nos sintamos felices en conjunto (p. 152).

Finalmente, es importante señalar entonces que el vínculo entre el fluir y la felicidad depende de la actividad en la cual la persona se involucre y de si esta actividad es capaz de conducirla a nuevos retos y desafíos que impliquen un crecimiento personal y cultural (Csikszentmihalyi, 1996/1998).

\section{Conclusiones}

A lo largo de este trabajo se ha podido constatar la complejidad del fenómeno de la creatividad y la cantidad de abordajes posibles para su estudio. Incluso, lo que se ha expuesto, son tan sólo algunas ideas y posibles explicaciones, de todo el conjunto existente en la literatura referente al tema. Los aportes y/o perspectivas desarrolladas, intentan explicar, como ya se ha visto, las relaciones existentes entre psicopatología y creatividad. Todos los autores mencionados, de las diferentes corrientes teóricas desde las cuales sostienen sus trabajos, están de acuerdo en decir que no existe una relación de tipo causal entre la psicopatología y la creatividad. Sin embargo, todos acuerdan en que sí existe algún tipo de relación entre ambos conceptos.

Como algunos estudios señalan, la creatividad entraña en sí misma algunos riesgos potenciales, como ser: el estilo cognitivo involucrado, el ámbito en el cual se desarrolle la actividad creativa, la idiosincrasia dominante de determinados ámbitos creativos y la posibilidad de permitir al sujeto el control sobre un aspecto de su realidad -la conciencia-. Todos estos riesgos están presentes, pero ninguno indica un diagnóstico certero de psicopatología. A su vez, cabe resaltar, que la literatura existente sobre creatividad indica claramente que su asociación a la psicopatología no es independiente del ámbito o dominio en el cual ésta se desarrolle. Como los estudios de Ludwig (1998), Becker (2000-2001) y Simonton (S.f.) señalan que los individuos pertenecientes a ámbitos artísticos tienden a presentar una mayor tasa de prevalencia de trastornos mentales que los individuos pertenecientes a ámbitos científicos. Estos estudios dejan entrever que la creatividad por sí sola, no conduce a una condición psicopatológica, sino que depende de múltiples factores la conformación de un cuadro psicopatológico. 
En consonancia con lo anterior, al revisar los aportes de la psicometría y la psicología cognitiva, referentes a la asociación entre psicopatología y creatividad, se encontró que los individuos altamente creativos presentaban un mayor riesgo a padecer de algún tipo trastorno psicológico (Simonton, S.f.). Sin embargo, de acuerdo a estos resultados, quedaría pendiente la evaluación de si el riesgo de padecer psicopatología en los sujetos creativos dedicados al ámbito artístico se da sólo en aquellos sujetos que presentan índices muy altos de creatividad, o si el nivel de creatividad presentado es independiente de la presencia o ausencia de psicopatología asociada.

Por otro lado, se ha señalado en la introducción de este trabajo la importancia de la creatividad para el desarrollo de una sociedad y para el progreso científico, pero queda claro, luego de revisar los aportes de la Psicología Positiva, la importancia que esta tiene para el mejoramiento de la calidad de vida y el crecimiento de la persona (Csikszentmihalyi, 1990/1997, 1996/1998). A pesar de los riesgos mencionados, la creatividad permite tanto integrar diferentes aspectos de la personalidad, mejorar el autoconcepto que las personas tienen de sí mismas (Franco Justo, 2004), así como también, disfrutar de distintas actividades que involucran y actualizan todas nuestras potencialidades.

También se reconocen las limitaciones existentes respecto de los instrumentos utilizados en la medición del constructo de la creatividad, y del constructo de la personalidad. Es un constante desafío para la investigación, lograr confeccionar instrumentos lo más adecuados posibles para la medición de los constructos mencionados. Sin embargo, muchos progresos se han realizado en este sentido desde los primeros aportes de Guilford en la década del '50. Se han desarrollado muchas otras técnicas de pensamiento divergente (Artola, Ancillo, Mosteiro y Barraca, 2004; Corbalán Berná, Martínez Zaragoza, Donolo, Alonso Monreal, Tejerina Arreal y LImiñana Gras, 2003; Torrance, 1966), así como también escalas de personalidad creativa (Garaigordobil y Pérez, 2005; Weschler, 1999), escalas de estilos de pensamiento (Sternberg, 1997; Weschler, 2006), todos instrumentos pertinentes en el ámbito de la evaluación de la creatividad.

Para finalizar, y respondiendo la pregunta inicial que dio lugar a este trabajo, se podría decir que la creatividad es un riesgo pero también un recurso. Es decir, la persona tiene la posibilidad de utilizar este recurso para perseguir su propio bien y el de la sociedad en su conjunto. Sin embargo, como se ha visto, ninguna actividad, facultad o habilidad, dejada a su libre curso o fluir es saludable para el desarrollo de la persona en su totalidad. En este sentido, la visión de los iluministas, respecto de la necesidad de un equilibrio entre las facultades y habilidades mentales para el bienestar personal, sigue siendo, hasta el día de hoy, un requisito necesario. Además, subrayamos la importancia de que este recurso, dispuesto no sólo al bien personal sino también al bien común, pueda ser una fuente de satisfacciones y logros para cualquier ser humano implicado en las problemáticas sociales que le rodean. 
La investigación sobre las posibles relaciones entre creatividad y psicopatología sigue abierta. Este trabajo no pretende cerrar el debate, si no más bien, brindar algunos datos que sirvan para continuar la discusión y ampliarla hacia otros interrogantes aún no explorados.

\section{Referencias Bibliográficas}

Artola, T., Ancillo, I., Mosteiro, P. y Barraca, J. (2004). PIC. Prueba de Imaginación Creativa. Madrid: TEA, Ediciones.

Becker, G. (2000-2001). The association of creativity and psychopathology: its cultural-historical origins. Creativity Research Journal, 13(1), 45-53.

Camacho, J. M. (2005) El humor y la dimensión creativa en psicoterapia. Psicodebate. Psicología, cultura y sociedad, 5(1), 45-58. Facultad de humanidades y ciencias sociales. Universidad de Palermo.

Carr, A. (2007). Psicología positiva. La ciencia de la felicidad. (G. Sánchez Barberán Tr.). Barcelona: Paidós. (Trabajo original publicado en 2004).

Claridge, G. (1972). The schizophrenias as nervous types. British Journal of Psychiatry, 121, 1-17.

Contini de González, N. (2000) La creatividad como recurso de afrontamiento en la vida cotidiana. Psicodebate. Psicología, Cultura y Sociedad, 1(1), 19-25. Facultad de humanidades y ciencias sociales. Universidad de Palermo.

Corbalán Berná, F. J., Martínez Zaragoza, F., Donolo, D. S., Alonso Monreal, C., Tejerina Arreal, M. y LImiñana Gras, R. M. (2003). Inteligencia creativa. CREA, Manual. Madrid: TEA Ediciones.

Csikszentmihalyi, M. (1997). Fluir. Una psicología de la felicidad. (N. López Tr.). Buenos Aires: Kairos. (Trabajo original publicado en 1990)

Csikszentmihalyi, M. (1998). Creatividad. El fluir y la psicología del descubrimiento y la invención (J. P. Tosaus Abadía Tr.). Buenos Aires: Paidós. (Trabajo original publicado en 1996)

Eysenck, H. J. (1993). Creativity and personality: suggestions for a theory. Psychological Inquiry, 4(3), 147-178.

Eysenck, H. J. (1994). Creativity and personality: word association, origins and psychoticism. Creativity Research Journal, 7, 209-216.

Eysenck, H. J. (1995). Genius: the natural history of creativity. Cambridge, England: Cambridge University Press. 
Franco Justo, C. (2004). Aplicación de programa psicoeducativo para fomentar la creatividad en la etapa de educación infantil. Relieve, 10 (2), 245-266.

Garaigordobil, M. y Pérez, J. I. (2005). Escala de personalidad creadora: estudio psicométrico exploratorio. Estudios de Psicología, 26(3), 345-364.

Guilford, J. P. (1956). The structure of the intellect. Psychological Bulletin, 53, 267-293.

Guilford, J. P. (1968). Intelligence, creativity and their educational implications. San Diego, California: Robert R. Knapp.

Judge, T.A., Erez, A., Bono, J. E. y Thoresen, C.J. (2002). Are measures of self-esteem, neuroticism, locus of control, and generalized self-efficacy indicators of common core construct? Journal of Personality and Social Psychology, 83, 693-710.

Ludwig, A. M. (1992) Creative achievement and psychopathology: Comparisons among professions. American Journal of Psychotherapy, 46, 330-356.

Ludwig, A. M. (1995). The price of greatness: resolving the creativity and madness controversy. New York: Guilford.

Ludwig, A. M. (1998). Method and madness in the arts and sciences. Creativity Research Journal, 11(2), 93-101.

Mandelbrot, B. (1988). Fractal geometry of nature. New York: W. H. Freeman. Martinez, C. (S.f.). Creatividad: recurso o insumo?... vital. Extraído el 3 de junio de 2009 desde: http://www.suicidologia.org.ar/textos.php.

Marietán, H. R. (1996). Curso de semiología psiquiátrica: funciones básicas. Buenos Aires: Ananké.

McConaghy, N. (1960). Modes of abstract thinking and psychosis. American Journal of Psychiatry, 114, 106-110.

McConaghy, N. (1961). The measurement of an inhibitory process in human higher nervous activity: Its relation to allusive thinking and fatigue. American Journal of Psychiatry, 118, 125-132.

McConaghy, N. \& Clancy, M. (1968). Familial relationship of allusive thinking in university students and their parents. British Journal of Psychiatry, 114, 1079-1087.

Ornstein, R. (1997). The right mind. New York: Harcourt Brance.

Peterson, J. B. \& Carson, S. (2002). Latent inhibition and openness to experience in a high-achieving student population. Personality and Individual Differences, 28, 323 -332.

Peterson, J. B., Smith, K. W. \& Carson, S. (2002). Openness and extraversion are associated with reduced latent inhibition: replication and commentary. Personality and Individual Differences, 33(7), 1137-1147. 
Prenda, K. M. y Lachman, M. E. (2001). Planing for the future: a life Management strategy for increasing control and life satisfaction in adulthood. Psychology and Aging, 16, 206-216.

Prentky, R. A. (2000-2001). Mental illness and roots of genius. Creativity Research Journal, 13(1), 95-104.

Rey, F. G. y Martinez, A. M. (1989) La personalidad: su educación y desarrollo. Cuba: Editorial Pueblo y Educación.

Simonton, D. K. (S.f.) Creativity and psychopathology. Contemporary answers to an ancient question. Obtenido 31 de mayo de 2009 en: http:// psychology.ucdavis.edu/simonton/samplepaper175.pdf

Simonton, D. K. (2005) Are genius and madness related? Psychiatric Times, 22 (7). Obtenido 16 de junio de 2009 en: http://www.psychiatrictimes.com/display/ article/10168/52456?pageNumber $=3$

Sternberg, R. J. (1997). Thinking styles. Cambridge: University of Cambridge.

Sternberg, R. J. y Lubart, T. I. (1997). La creatividad en una cultura conformista. Un desafío a las masas. (Ferran Meler Tr.). Barcelona: Editorial Paidós. (Trabajo original publicado en 1995).

Torrance, E. P. (1966). Torrance tests of creative thinking. Norms and technical manual. Lexington: M. A. Personnel Press.

Wasylkiew, L. y Fekken, C. G. (2002). Personality and self-reported health: Matching predictors and criteria. Personality and Individual Differences, 33, 607-620.

Weschsler, S. M. (1999). Avaliação multidimensional da criatividade: uma realiadade necessária. Extraído el 22 de mayo de 2009 desde: http:// www.potencializar.com.br/Publicacoes.htm.

Weschsler, S. M. (2006). Estilos de pensar y criar: impacto nas areas educacional y professional. Psicodebate. Psicología, cultura y sociedad, 7, 207-218. Facultad de humanidades y ciencias sociales. Universidad de Palermo.

Weschsler, S. M. y Nakano, T. C. (2002). Caminhos para a avaliação da criatividade: perspectiva brasileira. Em R. Primi (org.). Temas em Avaliação Psicológica. Campinas: IDB (pp. 103- 115).

Wikipedia. Enciclopedia de contenido libre mantenida por la Fundación Wikipedia sin fines de lucro. (http://es.wikipedia.org/wiki/Wikipedia: Portada). 
